\title{
Éducation Relative À L'environnement En Milieu Scolaire Ivoirien : Réflexion Pour Un Regard Critique
}

\author{
Dr. Brou Ahossi Nicolas
}

Enseignant-Chercheur, UFR - Sciences de l'Homme et de la Société (SHS),

Institut des Sciences Anthropologiques de Développement (ISAD)

Université Félix Houphouët-Boigny, Abidjan, (Côte d’Ivoire)

doi: 10.19044/esj.2016.v12n2p389 URL:http://dx.doi.org/10.19044/esj.2016.v12n2p389

\begin{abstract}
Environmental education uses some means and methods in order to create an environmental consciousness to the population. However, one seems to ignore the different hindrances engendered by the lack of appropriate teaching materials. The weight and influence of society and culture are perceived as potential obstacles. Our work aims at drawing people's attention on what can be regarded as essential in education in connection with the environment of Côte d'Ivoire such as school environment. In our work, we will focus on the content and the methods of the teachings of this specific domain. In so doing, we will have a glance on primary school boys and their teachers' behaviors and attitudes. This will help us to see the relationship they establish between "Health and Environment". The methodology will revolve around the analysis of the content of the teachings and on a qualitative and quantitative study. Primary school boys and their teachers is target population of the survey.

At the end of our analysis, it appears that the content of teachings linked to environment is not so explicit. The teaching method is suffering from a lack of explicit didactic and pedagogic materials. From the viewpoint of learns and teachers, we note a paradox between their words and deeds concerning environment. Finally, the link the learners and their teachers establish between Health and Environment let us see some problems of health. We wonder if people are aware of the threats and the social representations of environment in school zone for a sustainable development of our society. This is what can be a logic outcome of work.
\end{abstract}

Keywords: Environmental education, primary school, sustainable development. 


\section{Résumé :}

L'Education relative à l'Environnement (ERE) met en avant des moyens et méthodes pour donner une conscience Environnementale à la population. Il semble cependant que l'on feint d'ignorer bon nombre de difficultés dont l'insuffisance ou la vétusté des matériels didactiques. Des pesanteurs socioculturelles apparaissent aussi comme obstacles potentiels.

Notre réflexion a pour objectif de jeter un regard qui se veut critique sur l'éducation relative à l'environnement en Côte d'ivoire, notamment en milieu scolaire. Il s’agit d'apprécier le contenu et les méthodes des enseignements dispensés en matière d'ERE, d'appréhender les attitudes et comportement des élèves du primaire et de leurs enseignants, d’apprécier également le lien qu’ils établissent entre la « santé et l'environnement ».

La méthodologie s'articule autour de l'analyse de contenu des enseignements et d'une étude à la fois qualitative et quantitative. Des élèves du primaire et leurs enseignants constituent la population d'enquête.

Au terme de notre réflexion, il apparaît que le contenu des enseignements relatifs à l'environnement ne semble pas suffisamment explicite. La méthode d'enseignement souffre d'une insuffisance de matériels didactiques et pédagogiques assez explicites. Entre le dire et l'agir des élèves et enseignants, l'on note un paradoxe en lien avec leurs attitudes et comportements en matière d'environnement. Enfin, si le lien fait par les élèves et enseignants entre santé et environnement mentionne les problèmes de santé, l'on s’interroge sur la connaissance réelle des risques encourus et des représentations sociales de l'environnement en milieu scolaire pour un développement durable de nos sociétés. Tel pourrait être une suite logique à notre réflexion.

Mots-clés : Education relative à l'Environnement, Ecole primaire, Développement Durable

\section{INTRODUCTION}

«Le monde actuel prend peu à peu conscience des défis auxquels il se verra confronter dans un futur proche, à savoir l'épuisement des ressources naturelles et la sauvegarde de l'environnement. Ces préoccupations ont conduit progressivement au concept de Développement Durable (DD) qui se traduit par l'amélioration qualitative d'un état initial à travers des mesures structurantes, des comportements et surtout un mode de gouvernance qui place l'homme et sa qualité de vie au centre des politiques publiques. Agir en vue d'un développement durable, c'est fonder une vision du monde sur la base d'une éthique de la responsabilité et de la solidarité, au niveau local et mondial, en respectant le cadre des droits humains et celui des bases de la vie, afin de parvenir à une forme de développement durable. 
Pour être admis comme processus, le développement durable doit être connu, reconnu de tous et engager tous les acteurs de la société. L'un des moyens les plus sûrs pour y parvenir est l'éducation qui résulte d'une formation adéquate, de qualité et adaptée aux réalités sociales, culturelles, économiques et religieuses». ${ }^{26}$

La présente réflexion a pour enjeux de porter un regard critique sur l'Education Relative à l'Environnement en milieu scolaire ivoirien. L'objectif est de contribuer à l'appréciation du type d'éducation relative à l'environnement enseigné dans des établissements primaires publics ivoiriens.

De manière spécifique, il s'agit de :(i) apprécier le contenu et les méthodes des enseignements dispensés en matière d'ERE,(ii) appréhender les attitudes et comportement des élèves du primaire et de leurs enseignants et (iii) apprécier également le lien qu'ils établissent entre "Santé et Environnement ".

Dans les paragraphes qui suivent, après le contexte/problématique, nous allons présenter la méthodologie de l'étude suivie des résultats obtenus avant la discussion puis la conclusion.

\section{I- CONTEXTE/PROBLEMATIQUE}

Le développement durable se veut un processus de développement qui concilie l'écologique, l'économique et le social et établit un cercle vertueux entre ces trois pôles : C’est un développement, économiquement efficace, socialement équitable et écologiquement soutenable. Il est respectueux des ressources naturelles et des écosystèmes, support de vie sur Terre, qui garantit l'efficacité économique, sans perdre de vue les finalités sociales du développement que sont la lutte contre la pauvreté, contre les inégalités, contre l'exclusion et la recherche de l'équité. Une stratégie de développement durable doit être gagnante de ce triple point de vue, économique, social et écologique.

L'Education au Développement Durable (E.D.D.) ${ }^{27}$ répond aux objectifs du Grenelle Environnement qui a souligné la nécessité de renforcer la dimension éducative d'une politique globale de développement durable. En effet, "L'E.D.D. doit être une composante importante de la formation initiale des élèves, dès leur plus jeune âge et tout au long de leur scolarité, pour leur permettre d'acquérir des connaissances et des méthodes nécessaires pour se situer dans leur environnement et y agir de manière responsable. »

\footnotetext{
${ }^{26}$ http://www.globaleducation.ch/globaleducation_fr/resources/XY/DefEDD_FEDFEE.pdf

${ }^{27}$ La décennie de l'Education en vue du Développement Durable (DEDD) est une initiative de l'UNESCO pour promouvoir davantage et favoriser la prise en compte du Développement Durable dans les programmes d'enseignement.
} 
Ainsi, l'enseignement qu'il soit du niveau primaire, secondaire ou supérieur, est aussi un opérateur essentiel pour former au développement durable les dirigeants ainsi que la population civile et active de demain.

Pour Pascal HOUENOU ${ }^{28}$ « L'EDD devrait permettre à chaque être humain d'acquérir des connaissances, des compétences, des attitudes et des valeurs en lien avec le développement durable. » sont concernées par l’EDD, toutes les formes d'éducation formelle, non formelle, informelle, petite enfance, primaire, Secondaire, supérieure, y compris l'alphabétisation fonctionnelle.

Face à cette panoplie d'observation et de discours sur le développement durable, Lucie Sauvé (2000) insiste quant à elle, sur une composante fondamentale de l'EDD, celle de l'Education Relative à l'Environnement (ERE).

En effet, selon elle, l'ERE participe d'une éducation apte à promouvoir un développement humain intégral. ${ }^{29}$

Selon Aboua, $2005^{30}$, «l'éducation environnementale apparaît comme une nécessité dans la problématique du développement durable tant il apparaît que rien ne changera sans une réelle appropriation du concept de développement durable par les populations. Ainsi, l'éducation environnementale se positionne comme un moyen d'insertion des problèmes d'environnement et de développement dans la culture. Dans cette optique, l'éducation environnementale constitue une véritable force en favorisant la maturation d'un nouveau type de citoyen informé, sensibilisé et engagé, apte à résoudre les problèmes à travers des décisions enrichies par le processus participatif (démocratique).

Malgré la reconnaissance de l'importance cruciale de l'éducation environnementale dans la promotion du développement durable (Chapitre 36, Agenda21 ), peu de progrès ont été réalisé dans le domaine : les systèmes d'éducation formelle nationaux ont très peu évolué depuis Rio quant à la prise en compte de la dimension environnementale ; les projets d'éducation à l'environnement restent encore l'apanage d'ONG et d'individus sans soutien

\footnotetext{
${ }^{28}$ Prof. Pascal HOUENOU, Doyen honoraire de l'UFR SGE, Membre du Groupe mondial de référence de l'UNESCO pour l'EDD

${ }^{29}$ SAUVE Lucie, L'éducation relative à l'environnement entre modernité et postmodernité. Les propositions du développement durable et de l'avenir viable, Université du Québec à Montréal, (www.centrere.uqam.ca/public html/pdf/publication/ERE4.pdf) In A. Jarnet, Jickling, B., L. Sauvé, Arjen Wals et Priscilla Clarkin (dir.). (2000). The Future of Environmental Education in a Postmodern World? Whitehorse: Canadian Journal of Environmental Education, p57-71.

30 ABOUA Gustave, «L'éducation au service de l'environnement, le socle du développement durable en Afrique », in Actes du Colloque "développement durable, leçons et perspectives », Ouagadougou, 2004.
} 
politique suffisant ; d’ailleurs, les moyens financiers mis en œuvre pour la promotion de l'éducation environnementale restent insuffisants. »

La Côte d'Ivoire ne reste en marge de la question du Développement Durable ni particulièrement celle de l'Education relative à l'Environnement. En effet, la politique de l'éducation en Côte d'Ivoire est conduite à travers différents ministères. Chaque ordre d'enseignement correspond plus ou moins à un ministère autonome. L'enseignement primaire, qui nous intéresse, est du ressort du Ministère de l’éducation nationale. Depuis 1993, en collaboration avec le ministère de l'Éducation nationale, la Banque Africaine de Développement (BAD) met en place un projet dit « Projet BAD éducation IV » pour améliorer la qualité de l'enseignement.

Parler justement de qualité de l'enseignement, une question particulière, au cœur de notre réflexion nous invite à réfléchir sur la place de l'Education Relative à l'Environnement comme socle d'un Développement Durable.

\section{II- METHODOLOGIE}

Comme toute étude scientifique, une démarche méthodologique a été adoptée. Le choix du site et de la population d’étude a été la première action posée.

\section{1- Champ géographique et sociologique}

La collecte des données s’est effectuée à Abidjan, précisément au sein de deux (2) écoles primaires de la commune de Yopougon. Le choix s'est portée sur la commune de Yopougon compte tenue de la diversité des écoles qu'elle abrite et surtout de la réponse positive obtenue rapidement auprès de l'Inspection de l'Enseignement Primaire I pour réaliser l'étude dans les écoles primaires retenues.

Les deux écoles ont été choisies dans deux quartiers différents, l’une résidentielle (Groupe scolaire de GFCI) et l'autre de moyen-standing (Groupe scolaire Mosquée de Wassakara).

Les élèves des écoles primaires choisis représentent le groupe social de la population de l'étude. Nous avons pris pour cible principalement un échantillon issu des élèves des classes du Cours Elémentaire $2^{\text {ème }}$ année (CE2) et du Cours Moyen $2^{\text {ème }}$ (CM2). Chaque classe a vu l'enseignant titulaire être systématiquement retenu comme personne ressource dans la population d'étude. Nous avons retenu initialement 60 élèves soit 30 par établissement. Cependant, après dépouillement des questionnaires, 53 élèves soit 27 au Groupe scolaire de GFCI et 26 Groupe scolaire Mosquée de Wassakara ont vu leur questionnaire validé. Les Directeurs des 2 écoles primaires ont également été retenus comme acteurs-clé. 


\section{2- Collecte de données}

Une recherche documentaire nous a permis de mieux cerner l'objet de la présente réflexion.

Il a été question de collecter des informations générales et particulières relatives aux méthodes, modes et pédagogies matière d'enseignement relatif à l'environnement.

Les recherches se sont effectuées dans différentes bibliothèques ou centres de documentation à Abidjan aussi bien dans les écoles primaires et à l'Inspection de l'Enseignement primaire; D'autres recherches ont été effectuées dans les bibliothèques de l'Institut de Recherche pour le Développement (IRD), Centre de Recherches pour l'Action et la Paix (CERAP) anciennement appelé INADES ainsi que à l'Institut d'EthnoSociologie (I.E.S) et à la bibliothèque centrale de l'Université Félix Houphouët-Boigny (Cocody).

Les techniques et outils d'enquêtes utilisées sont l'enquête de terrain à l'aide d'un questionnaire adressé aux élèves ainsi que des entretiens semidirectifs avec comme support, un guide d'entretien.

En appui à l'enquête et aux entretiens, nous avons également utilisé une grille d'observation lors de notre passage dans les deux (2) établissements scolaires choisis.

L'enquête par questionnaire comportait les caractéristiques de l'enquêté. Il avait pour but de recueillir des informations relatives à la connaissance des élèves en lien avec l'environnement et leurs appréciation des modules enseignés ainsi que des méthodes et matériels didactiques utilisés par leurs enseignants.

Les entretiens individuels se sont focalisés sur les enseignants des classes retenues ainsi que les directeurs des 2 écoles primaires. L'entretien a abordé les modules de formations, la pédagogie et le matériel didactiques ainsi que les recommandations.

Les méthodes d'analyse des données utilisées étaient l'analyse de contenu et l'analyse statistique.

Enfin, une grille d'observation a permis d'observer les salles de classes, les cours de récréation ainsi que les abords immédiats des écoles.

Les données ainsi collectées ont été traitées manuellement pour les entretiens et la grille d'observation. Quant aux données du questionnaire, elles ont été traitées grâce au logiciel Epiinfo 6.04.

Il revient à présent à exposer les résultats de l'étude avant de les discuter.

\section{III- RESULTATS}

Quelle est la place de l'ERE dans les programmes d'enseignement dans les écoles primaires publics ivoiriens? Comment l'ERE est-elle enseignée ou dispensée par les enseignants? Quels sont les outils 
pédagogiques et didactiques utilisés ? Quels sont les attitudes et comportements des élèves du primaire et de leurs enseignants vis-à-vis de la question de l'environnement et comment ou quel lien établissent-ils entre Environnement et Santé ? Autant de questions qui ont orienté notre étude dont nous exposons les résultats obtenus.

\section{1 - Brèves caractéristiques de l'échantillon}

Comme indiqué dans la méthodologie, notre échantillon se compose des élèves du primaire ainsi que de leurs différents enseignants et des directeurs d'établissements, soit un total de 53 élèves, 4 enseignants et 2 directeurs.

Les tableaux ci-après nous indiquent la taille de l'échantillon, au niveau des élèves, par école et par classe.

TABLEAU 1 : TAILLE DE L'ECHANTILLON PAR ECOLE

\begin{tabular}{|c|c|c|}
\hline & Nbre & $\%$ \\
\hline Groupe scolaire GFCI & 27 & 50,94 \\
\hline Groupe scolaire MOSQUEE & 26 & 49,06 \\
\hline Total & 53 & 100 \\
\hline
\end{tabular}

Source : Notre enquête

TABLEAU 2 : TAILLE DE L'ECHANTILLON PAR CLASSE

\begin{tabular}{|c|c|c|c|}
\hline & & Nbre & $\%$ \\
\hline \multirow{3}{*}{ Groupe scolaire GFCI } & CE2 & 12 & 22,64 \\
\hline & CM2 & 15 & 28,30 \\
\hline & Sous-Total & 27 & 50,94 \\
\hline \multirow{3}{*}{ Groupe scolaire MOSQUEE } & CE2 & 12 & 22,64 \\
\hline & CM2 & 14 & 26,42 \\
\hline & Sous-Total & 26 & 49,06 \\
\hline Total & & 53 & 100 \\
\hline
\end{tabular}

Source : Notre enquête

3. 2 - Du contenu et les méthodes des enseignements dispensés en matière d'ERE

L'environnement, pris comme matière spécifique n’est pas enseigné dans les différents établissements primaires de l'étude. Les propos recueillis auprès des enseignants est évocateur de la réalité observée: «nous avons des disciplines telle que la Géographie ou encore les Sciences de la nature que nous enseignons aux élèves; c'est pendant ces cours que nous leur expliquons ce qu'est l'environnement..... »

Les méthodes employées sont quasiment les mêmes dans les deux établissements. Les enseignants utilisent le tableau noir et des planches à dessin s’il en existe pour illustrer leurs propos. Les élèves rencontrés ont énoncé une série de matériels didactiques que leurs différents maitres de 
classes utilisent. Ce sont entre autres, les livres de classe, notamment le livre de Sciences et Technologie.

"La maîtresse nous montre l'environnement dans le livre de Sciences " nous dit cet élève de CM2 au groupe scolaire GFCI. " Il y'a des jours où le maître dessine au tableau » nous dit cet autre élève de CE2 au groupe scolaire Mosquée.
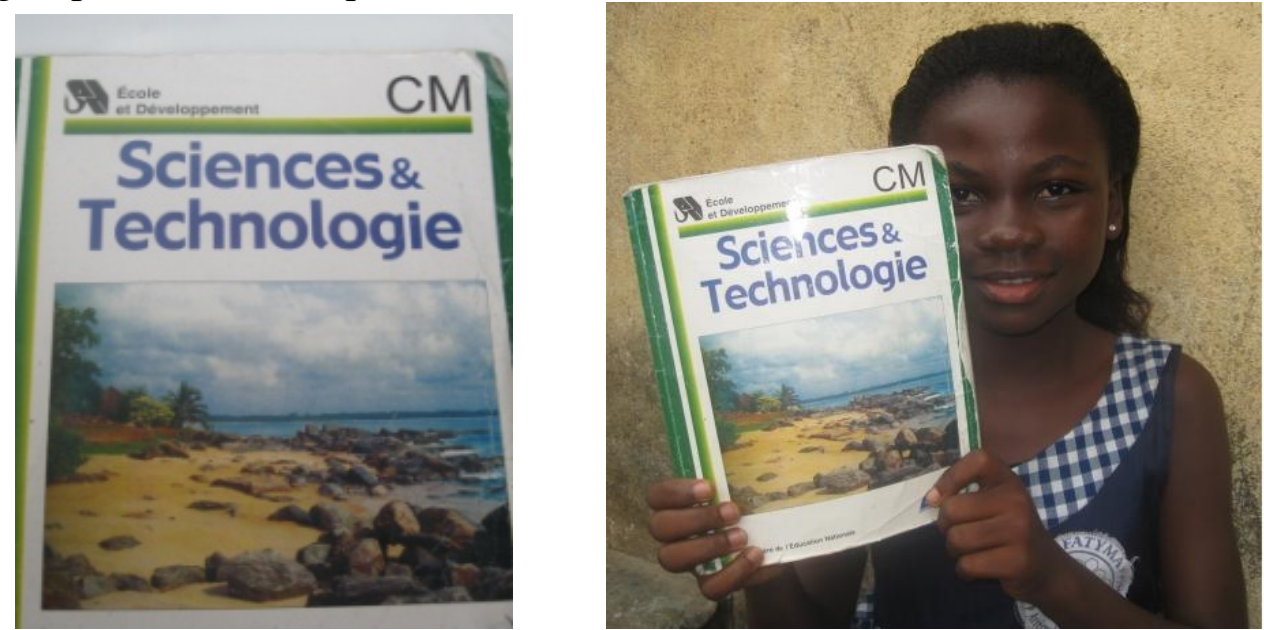

PHOTO 1 : UN LIVRE DE SCIENCES ACTUELLEMENT UTILISE EN CLASSE DE CM (1\&2)
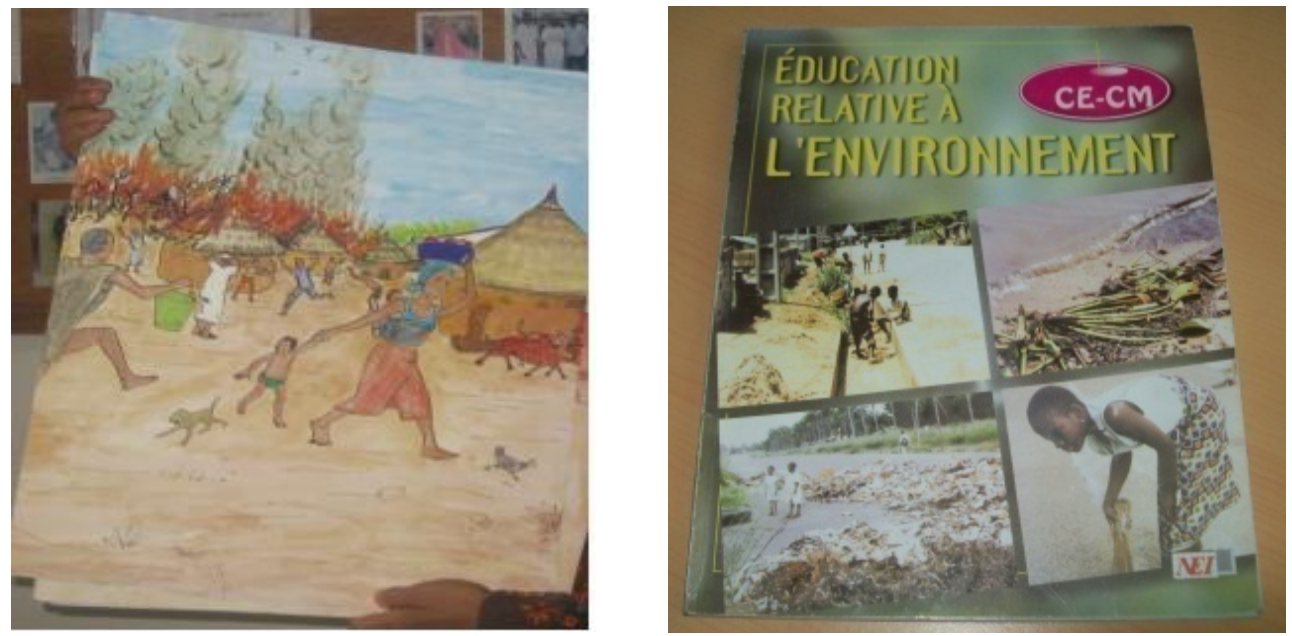

PHOTO 2 : UNE PLANCHE DE SENSIBILISATION PHOTO 3: UN LIVRE D'ERE NON ENCORE VULGARISE

\section{3 - Attitudes et comportement des élèves du primaire et de leurs enseignants}

$\mathrm{Au}$ cours de nos différents passage au sein des établissements d'enquêtes, nous avons procédé par observation afin d'identifier les actes posés par les élèves et le corps enseignants en relation avec «leur cadre de 
travail » notamment, les salles de classe, la cours de récréation et les toilettes des établissements. Si les salles de classes sont bien entretenues dans l'ensemble, la cour de récréations et les toilettes le sont moins.

Les maîtres d'écoles organisent des séances de balayages des salles de classes à tour de rôle, par les leurs élèves respectifs. Parfois, c'est une opération de nettoyage générale ou d'entretien de la cours qui est organisée. ".....nous essayons de faire comprendre aux élèves, le sens de la propreté » nous dit cet enseignant du groupe scolaire GFCI.

"Les enfants sont souvent les premiers responsables de l'état des toilettes. On organise des séances de nettoyage mais à chaque fois, les plus petits élèves surtout, salissent les murs ou même le sol par leurs selles ou urines «nous dit un Directeur d'établissement. ".....et ce n'est pas facile avec les élèves. Ils mangent n'importent comment et jettent les bouts de papier ou les sachets plastiques dans la cour de récréation. Pourtant, nous avons mis des poubelles dans la cour de l'école », dit cet autre Directeur.

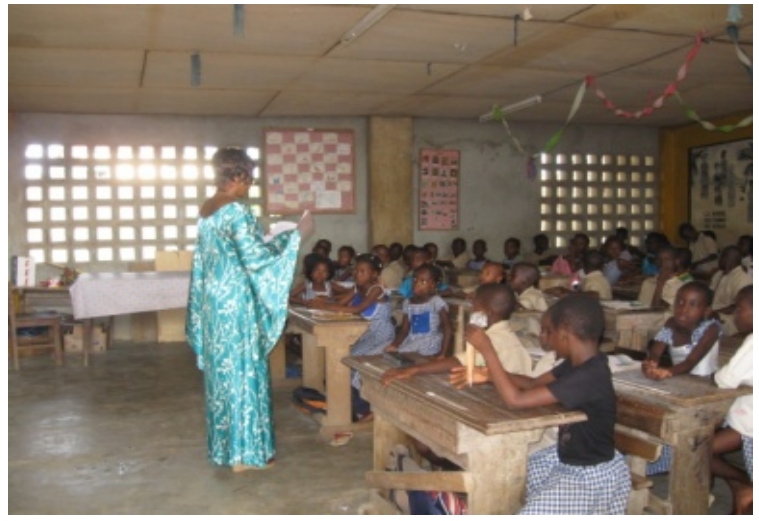

PHOTO 4 : UNE ENSEIGNANTE DU PRIMAIRE ET SES ELEVES DANS UNE SALLE DE CLASSE BIEN ENTRETENUE ET DECOREE
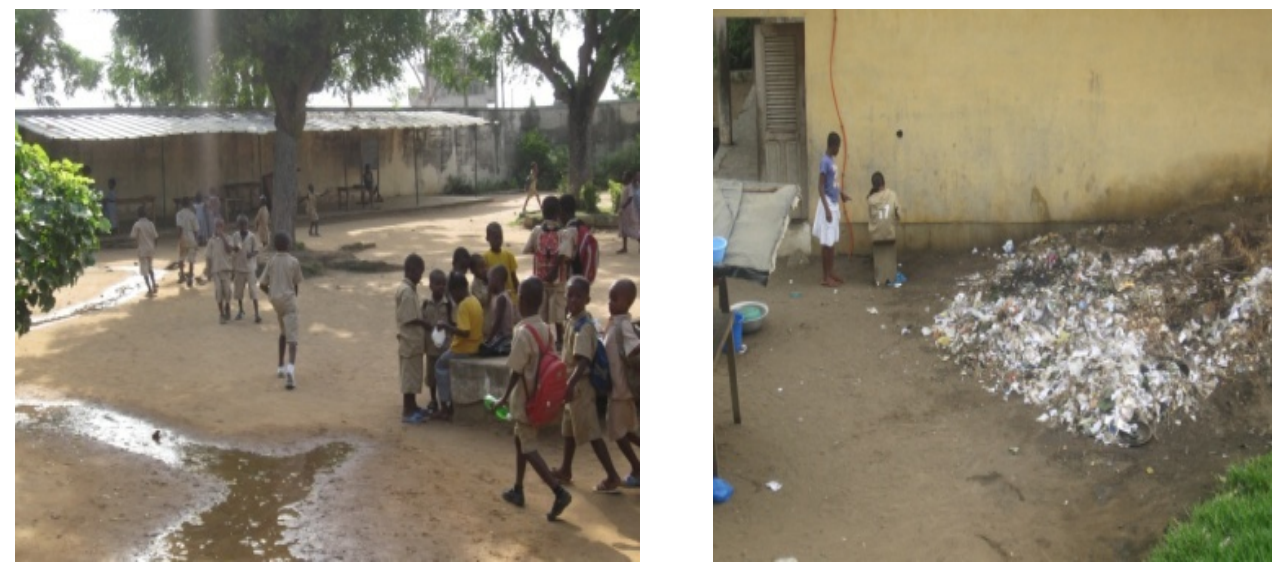

PHOTO 5: EAU DE RUISSELLEMENT ET TAS D'ORDURES DANS LA COUR DE L'ECOLE, PROCHE DE SALLES DE CLASSES 


\section{4 - Du lien "Environnement et Santé "}

Pour répondre à cette préoccupation, quatre questions ont été posées uniquement aux élèves :

- Qu'est-ce que l'environnement?

- Quels sont les éléments qui constituent l'environnement ?

- Qu'est ce qui peut détruire l'environnement ?

- Quels sont les problèmes posés par la destruction de l'environnement?

Les tableaux ci-après présentent les données recueillies.

\section{Qu'est-ce que l'environnement ?}

Les reponses a cette question posee uniquement aux eleves se trouvent . TABLEAU 3 : REPARTITION DES REPONSES PAR ITEMS

\begin{tabular}{|l|c|c|}
\hline \multicolumn{1}{|c|}{ Items de réponse } & Nbre & $\mathbf{\%}$ \\
\hline "Tout ce qui nous entoure " & 36 & 67,92 \\
\hline "C'est une invention" & 0 & 0 \\
\hline "C'est le milieu dans lequel on vit" & 4 & 7,547 \\
\hline "Autres" & 8 & 15,09 \\
\hline "Ne sais pas" (NSP) & 5 & 9,43 \\
\hline Total & 53 & 100 \\
\hline
\end{tabular}

Source : Notre enquête

TABLEAU 4 : REPARTITION DES REPONSES PAR ITEMS ET PAR NIVEAU D'ETUDE

\begin{tabular}{|l|r|r|r|c|}
\hline \multirow{2}{*}{ Classe } & \multicolumn{2}{|c|}{ CE2 } & \multicolumn{2}{c|}{ CM2 } \\
\cline { 2 - 5 } & Nbre & \% & Nbre & \% \\
\hline "Tout ce qui nous entoure " & 11 & 61,11 & 25 & 71,43 \\
\hline "C'est une invention" & 0 & 0 & 0 & 0 \\
\hline "C'est le milieu dans lequel on vit" & 0 & 0 & 4 & 11,43 \\
\hline "Autres" & 2 & 11,11 & 6 & 17,14 \\
\hline "Ne sais pas" (NSP) & 5 & 27,78 & 0 & 0 \\
\hline Total & 18 & 100 & 35 & 100 \\
\hline
\end{tabular}

Source : Notre enquête

\section{Commentaire des tableaux}

La lecture du tableau 3 montre la forte proportion de la Réponse 1. Selon les élèves enquêtés, l'environnement "Tout ce qui nous entoure " (67,92\%). C’est la définition généralement admise. Toutefois, les élèves ne manquent pas de souligner aussi que "C'est le milieu dans lequel on vit".

Au niveau des classes, dans le tableau 4, les élèves de CE2 et CM2 ont quasiment le même pourcentage, respectivement $61,61 \%$ et $71,43 \%$ des réponses. 


\section{$>$ Quels sont les éléments qui constituent l'environnement ?}

Les réponses à cette question sont aussi diverses que variées.

Si pour la majorité des élèves, l’environnement est, selon la définition qu'ils citent tous «Tout ce qui nous entoure », pour certains, les éléments constitutifs de cet environnement sont «..... les arbres, les animaux et l'eau » principalement. D’autres y ajoutent «....les maisons, les faune, les immeubles, les hommes, etc ».

\section{$>$ Qu'est ce qui peut détruire l'environnement ?} variées.

A cette question, une fois encore les réponses sont aussi diverses que

Cependant, la quasi-totalité des élèves ont évoqué «les feux de brousse " comme principale cause de destruction de l'environnement. Viennent ensuite "l'exploitation abusive de la flore et le braconnage ». Comme le dit cet élève de CM2 : «quand on coupe les arbres, on détruit l'environnement. Aussi, l'"assassinat" sauvage des animaux détruit l'environnement.

Certains ont évoqué le gaspillage de l’eau, la pollution, le rejet des ordures ménagères dans la rue, etc, autant d'actes qui nuisent à l’environnement.

Quels sont les problèmes posés par la destruction de l'environnement élèves.

Le tableau ci-après illustre l'ensemble des réponses proposées par les

TABLEAU 5 : REPARTITION DES REPONSES PAR ITEMS ET PAR NIVEAU D'ETUDE

\begin{tabular}{|l|r|r|r|r|r|r|r|}
\hline & \multicolumn{2}{|c|}{ CE2 } & \multicolumn{2}{c|}{ CM2 } & \multicolumn{2}{c|}{ Total } \\
\hline Items & Nbre & \multicolumn{1}{c|}{$\%$} & \multicolumn{1}{c|}{ Nbre } & \multicolumn{1}{c|}{$\%$} & \multicolumn{1}{c|}{ Nbre } & \multicolumn{1}{c|}{$\%$} \\
\hline "On peut tomber malade" & 15 & 75,00 & 20 & 60,61 & 35 & 66,04 \\
\hline "Les forêts vont disparaître" & 2 & 10,00 & 7 & 21,21 & 9 & 16,98 \\
\hline "Les océans vont augmenter" & 2 & 10,00 & 0 & 0 & 2 & 3,77 \\
\hline "Il pleut beaucoup pendant la saison sèche" & 0 & 0 & 1 & 3,03 & 1 & 1,89 \\
\hline "Il ne pleut plus pendant la saison des pluies" & 1 & 5,00 & 5 & 15,15 & 6 & 11,32 \\
\hline "Autres" & & & & & 0 & 0 \\
\hline "Ne sais pas" (NSP) & & & & & 0 & 0 \\
\hline Total & $\mathbf{2 0}$ & $\mathbf{1 0 0}$ & $\mathbf{3 3}$ & $\mathbf{1 0 0}$ & $\mathbf{5 3}$ & $\mathbf{1 0 0}$ \\
\hline
\end{tabular}

Source : Notre enquête

\section{Commentaire du tableau}

La lecture du tableau 5 montre la forte proportion de la Réponse 1. Selon les élèves enquêtés, si l'on détruit l'environnement "On peut tomber 
malade" (66,04\%). Les autres items de réponse sont proposés dans des proportions relativement faibles. "Les forêts vont disparaître" et "Il ne pleut plus pendant la saison des pluies" sont enregistrés avec respectivement $16,98 \%$ et $11,32 \%$.

Les résultats de notre étude ainsi présentés, il nous revient à présent d'aborder le dernier volet qu'est la discussion.

\section{IV- DISCUSSION}

Rappelons que l'Education Relative à l'Environnement est sujet de bon nombre d'écris. Notre étude vient pour essayer d'apporter un regard complémentaire en vue de poser une réflexion critique sur la pratique de l'enseignement de l'Education relative à l'Environnement

L’appréciation générale des résultats obtenus nous invite en effet à cette réflexion.

\section{1- Du contenu et de la méthode d'enseignement}

Si l'on s'en tient aux résultats obtenus, il apparait à l'évidence que l'Education Relative à l'Environnement est enseigné. Cependant, les différents programmes d'enseignement sur l'environnement proposés se résument en des "portions » d'informations distillés aux élèves lors de la dispensation d'autres matières. On retrouve la question de l'environnement, ou du moins, l'environnement est souvent abordé comme sujet lorsque l'enseignant dispense le cours de Sciences de la nature, ou encore de la géographie. D'autres aspects de la question sont abordés à travers le module de la Vie coopérative ou la pratique des activités d'embellissement et campagne de propreté.

Par ailleurs, il a été donné de constater que le matériel pédagogique semble vétuste voir insuffisant pour bien faire appréhender ou comprendre la question de l'Environnement aux élèves des classes primaires

Dessiner au tableau noir, ou montrer une planche à dessins ne semble pas assez parlant.

L'un des constats majeurs effectués au cours de l'enquête est notoirement celui de la fracture numérique et technologique. En effet, aucun des établissements choisis dans le cadre de l'étude ne possèdent de centre informatique, ni de salle informatique encore moins d'un ordinateur avec connexion Internet.

A l'ère du numérique, cela paraît surprenant mais démontre plutôt de l'urgence des autorités scolaires ou du pouvoir public à envisager un programme de dotation des établissements scolaires de centres informatiques et initier des programmes de formation en informatique et navigation (recherche sur les moteurs de recherche) dans le but d'emmener les élèves à 
s'auto-former en matière d'environnement voire de favoriser l'enseignement en ligne ou e-enseignement ${ }^{31}$.

Si pour Houenou ${ }^{32}$, les méthodes d'enseignement et d'apprentissage traditionnels semblent, il y'a lieu aujourd'hui d'adopter d'autres approches en vue d'un changement de comportement.

Certes, l'on peut réellement dire que l'inexistence de centre d'informatique est un obstacle à l'éducation relative à l'environnemental alors que des manuels et programmes sur l'environnement existent. Cependant, l'outil informatique serait d'un atout majeur pour prodiguer une éducation environnementale aux élèves. La projection de film-documentaire viendrait ainsi en complément à ce que les manuels, programmes et méthodes pédagogiques d'enseignement essaient tant bien que mal de faire, afin d'être plus efficace dans la quête de l'impact positifs sur les attitudes et comportement des élèves en lien avec la préservation de l'environnement. Comme le souligne Djané (2010), les documents et stratégies pédagogiques en classe ne constituent pas vraiment une démarche d'une éducation environnementale pour un développement durable. ${ }^{33}$

\section{2 - Des attitudes et comportements des élèves}

A l’issue des enquêtes de terrain réalisées dans les deux écoles primaires choisies, les résultats de l'étude montrent que malgré la dispensation des enseignements, les élèves, voire la cour de récréation des établissements n’échappent pas aux « mauvaises » habitudes.

L'arrière des salles de classes ou même un espace de la cour non aménagé sert de lieu de dépôts d’ordures ou des déchets en provenance de salles de classes. L'on assiste aussi à des fuites d'eau qui ruissellent en serpentant dans la cour de récréation.

Les enseignants semblent faire un choix de modules parfois en défaveur des modules sur l'environnement. L'état des cours d’école nous semble non seulement comme un paradoxe, mais bien plus comme une

\footnotetext{
${ }^{31}$ La formation en ligne ou encore l'apprentissage en ligne (au Canada), l'e-formation ou l'elearning, désignent l'ensemble des solutions et moyens permettant l'apprentissage par des moyens électroniques. La formation en ligne inclut ainsi des sites web éducatifs, la téléformation, l'enseignement télématique, ou encore l'e-training, notamment. La formation en ligne est une des technologies de l'information et de la communication pour l'éducation (TICE), intégrée dans la cyberculture.

${ }^{32}$ HOUENOU Pascal (2014), «L'éducation au développement durable en question » in Atelier de partage et de renforcement de capacités des medias francophones sur l'éducation pour le développement durable, Communication, Abidjan, du 14 au 15 Octobre 2014

${ }^{33}$ DJANE, K. Aristide (2010), Éducation environnementale à l'école ivoirienne: diagnostic et enjeux d'une pérennisation. Économie et management, 10 avril 2010. Disponible sur http://www.univtlemcen.dz/ fseg/Larevue10.htm
} 
conséquence de l’insuffisance de l’éducation environnementale due à la sélection de modules effectuée par les enseignants.

Soulignons que la langue d'enseignement est le français. C'est la langue officielle du pays. De fait, l’enseignement des différentes matières se fait en langue française. Initier des séances en langue locale (langue maternelle des élèves), malgré une bigarrure importante du fait de la grande variété d'ethnies en Côte d'Ivoire pourrait peut-être contribuer à la bonne appréhension de l'environnement.

\section{3 - Du lien fait par les élèves et enseignants entre santé et environnement}

Selon les élèves enquêtés, si l'on détruit l'environnement "On peut tomber malade" (66,04\%). Telle est l'un des pourcentages majeurs observés au cours de l'enquête.

Des efforts sont faits par le corps enseignants afin de d'entretenir les établissements cependant, beaucoup reste encore à faire.

A observer des dépôts d’ordures (déchets en provenance des salles de classe dans la cours de récréation, ou voir des ruissellements d'eau) pose le problème du lien santé et environnement. Une eau stagnante est source de lit pour le développement de l'anophèle par exemple. Et donc risques de maladies. Avoir un " dépotoir » au sein d’un établissement c’est également courir le risque d’occasionner des séries de maladies liées à l'hygiène.

Si dans le dire, les élèves et enseignants mentionnent bien qu'un environnement malsain peut provoquer des maladies, l'on se demande bien si le lien est évident ; car entre le dire et l'agir, il y’a malheureusement un fossé que l'on observe aisément.

\section{4 - Pour une approche novatrice multisectorielle et transdisciplinarité}

Une étude réalisée par DJANE $(2010)^{34}$ montre bien que « l'institutionnalisation de l'éducation environnementale à l'école ivoirienne souffre d'énormes insuffisances qui la mettent à mal » pour l'auteur, ces insuffisances sont (i) l'Absence de centre de recherche type aux questions d'éducation environnementale à l’école primaire, (i) l'absence de formations des enseignants (maîtres d'école) sur les questions environnementales et d'éducation environnementale. Par ailleurs, il note aussi la confusion entre l'éducation environnementale et l'éducation aux sciences (cette confusion s’observe également dans les ouvrages destinés aux élèves).

\footnotetext{
${ }^{34}$ DJANE, K. A. (2010). Éducation environnementale à l'école ivoirienne: diagnostic et enjeux d'une pérennisation. Économie et management, 10 avril 2010. Disponible sur http://www.univtlemcen.dz/ fseg/Larevue10.htm
} 
Il y a donc urgence de réorienter l'éducation en vue de bâtir un avenir meilleur pour tous. Ce qui requière la participation de tous, la motivation des apprenants et le Développement d'outils innovants et serait également source d'amélioration des conditions d'apprentissage et de formation en matière d'éducations relative à l'environnement.

Selon le Pr Houenou, l’Approche Ecosystème et Santé humaine ou "Ecosanté " pourrait être proposée comme piste novatrice d’Education Relative à l'Environnement afin de favoriser une Education qui est multisectorielle, multidimensionnelle et transdisciplinaire. «Depuis 1996, le CRDI appuie des projets de recherche visant à trouver des moyens d'améliorer la santé humaine et d'assainir les écosystèmes dans les pays en développement. Ces travaux ont contribué à l'établissement d'une nouvelle discipline, écosystèmes et santé humaine ou écosanté, tant sur le plan de la recherche, de la formation et des pratiques. L'écosanté est un moyen novateur et efficace de produire de nouvelles connaissances qui aideront à mieux s'attaquer aux compromis à faire entre moyens de subsistance, environnement et santé humaine» ${ }^{35}$

Ainsi l'approche écosanté consiste à mettre en évidence le lien Environnement et santé tout en impliquant plusieurs acteurs dans la production de connaissance scientifiques. Elle pourrait certainement contribuer à donner une meilleure éducation en matière d'environnement aux élèves.

\section{CONCLUSION}

Au terme de notre réflexion, il apparaît que l'enseignement dispensé en matière d'éducation relative à l'environnement en milieu scolaire ivoirien souffre de certaines insuffisances telles que présentés dans les résultats. Le contenu des enseignements en matière d'éducation relative à l'environnement ne semble pas suffisamment explicite. La méthode d'enseignement souffre d'une insuffisance de matériels didactiques assez explicites. Entre le dire et l'agir des élèves et enseignement l'on note un paradoxe en lien avec leurs attitudes et comportements en matière d'environnement. Enfin, si le lien fait par les élèves et enseignants entre santé et environnement mentionnent les problèmes de santé, l'on se doit de s'interroger sur la connaissance réelle des risques encourus et des représentations sociales de l'environnement en milieu scolaire. Tel pourrait être une suite logique à la présente réflexion.

Somme toute, l'ERE serait d'un atout vers un développement durable si elle est mieux dispensée et avec les moyens matériels et pédagogiques

\footnotetext{
${ }^{35}$ Source : site web du CRDI : www.crdi.org
} 
appropriés pour une mise en pratiques dans le quotidien des élèves du primaire.

\section{References:}

ABOUA Gustave (2004), «L'éducation au service de l'environnement, le socle du développement durable en Afrique ", in Actes du Colloque « développement durable, leçons et perspectives », Ouagadougou, 2004.

AKE A. Laurent et BONI, D (1990), Développement agricole et protection de la forêt: quel avenir pour la forêt ivoirienne ? Compte rendu de la XIIème réunion plénière de l'AETFAT, Hambourg (Allemagne), 1990.

DJANE K. Aristide. (2010), Éducation environnementale à l'école ivoirienne: diagnostic et enjeux d'une pérennisation. Économie et management, 10 avril 2010. Disponible sur http://www.univtlemcen.dz/ fseg/Larevue10.htm

HOUENOU P. Valentin (2014), « L’éducation au développement durable en question » in Atelier de partage et de renforcement de capacités des medias francophones sur l'éducation pour le développement durable, Communication, Abidjan, du 14 au 15 Octobre 2014

SAUVE Lucie (2000), L'éducation relative à l'environnement entre modernité et postmodernité Les propositions du développement durable et de l'avenir viable, Université du Québec à Montréal, (www.centrere.uqam.ca/public_html/pdf/publication/ERE4.pdf) In A. Jarnet, Jickling, B., L. Sauvé, Arjen Wals et Priscilla Clarkin (dir.). (2000). The Future of Environmental Education in a Postmodern World? Whitehorse : Canadian Journal of Environmental Education, p57-71.

UICN (UNION MONDIALE POUR LA NATURE) (2003), Rapport de synthèse de l'atelier sur la gouvernance des aires protégées d'Afrique, La Kompienga, (Burkina Faso), 2003. 